International Journal of Bifurcation and Chaos, Vol. 18, No. 11 (2008) 3221-3231

(C) World Scientific Publishing Company

\title{
ON THE DENSE ENTROPY OF TWO-DIMENSIONAL INHOMOGENEOUS CELLULAR NEURAL NETWORKS
}

\author{
JUNG-CHAO BAN* \\ Department of Mathematics, \\ National Hualien University of Education, \\ Hualien 97063, Taiwan \\ jcban@mail.nhlue.edu.tw \\ CHIH-HUNG CHANG \\ Department of Applied Mathematics, \\ National Chiao-Tung University, \\ Hsin-Chu 30050, Taiwan \\ grece.am92g@nctu.edu.tw
}

Received November 6, 2007; Revised December 10, 2007

\begin{abstract}
This investigation elucidates the dense entropy of two-dimensional inhomogeneous cellular neural networks (ICNN) with/without input. It is strongly related to the learning problem (or inverse problem); the necessary and sufficient conditions for the admissibility of local patterns must be characterized. For ICNN with/without input, the entropy function is dense in $[0, \log 2]$ with respect to the parameter space and the radius of the interacting cells, indicating that, in some sense, ICNN exhibit a wide range of phenomena.
\end{abstract}

Keywords: Entropy; learning problem; ICNN.

\section{Introduction}

Cellular neural networks (CNN), as presented by Chua and Yang [1988a, 1988b] have been extensively investigated, and described in a review [Chua, 1998], which contains relevant references. Two of their applications are in image processing and pattern recognition. An important class of applications is steady-state solutions, including mosaic solutions and defect solutions [Chua, 1998; Hsu et al., 2000; Juang \& Lin, 2000]. In recent years, the complexity of steady-state solutions has been extensively studied, and much attention has been paid to the complexity of the set of global patterns, with particular reference to entropy [Ban et al., 2001a, 2001b, 2002; Ban \& Lin, 2005; Ban et al., 2007a, 2007b; Chow et al., 1996a, 1996b; Hsu et al., 2000; Hsu \& Yang, 2002; Juang \& Lin, 2000; Lin \& Shin, 1999; Lin \& Yang, 2000, 2002; Lind \& Marcus, 1995].

Two-dimensional (2-D) CNN is of the form,

$$
\begin{aligned}
\frac{d x_{i, j}}{d t}= & -x_{i, j}+z+\sum_{|k|,|l| \leq d} a_{k, l} f\left(x_{i+k, j+l}\right) \\
& +\sum_{|k|,|l| \leq d} b_{k, l} u_{i+k, j+l},
\end{aligned}
$$

\footnotetext{
${ }^{*}$ This work is partially supported by the National Science Council, R.O.C. under Grant No. 95-2115-M-026-003.
} 
where $(i, j) \in \mathbb{Z}^{2}, d \in \mathbb{N}, f(x)$ is a piecewise-linear output function, defined by

$$
\begin{gathered}
y=f(x)=\frac{1}{2}(|x+1|-|x-1|) . \\
A=\left[a_{k, l}\right]=\left[\begin{array}{ccc}
a_{-d, d} & \cdots & a_{d, d} \\
\vdots & \ddots & \vdots \\
a_{-d,-d} & \cdots & a_{d,-d}
\end{array}\right] \text { and } \\
B=\left[b_{k, l}\right]=\left[\begin{array}{ccc}
b_{-d, d} & \cdots & b_{d, d} \\
\vdots & \ddots & \vdots \\
b_{-d,-d} & \cdots & b_{d,-d}
\end{array}\right]
\end{gathered}
$$

represent the feedback template and the controlling template, respectively; $z$ denotes the biased term or threshold. The quantities $x_{i, j}$ denote the state at cell $C_{i, j}$, and $y_{i, j}$ denote the output at $C_{i, j}$.

As is generally known, stationary solutions $\bar{x}=$ $\left(\bar{x}_{i, j}\right)$ are essential to understand CNN, and their outputs are called patterns. This study considers a specified class of output patterns called mosaic patterns. Ban et al. [2007b] investigated the connection between CNN with/without input and shift spaces, and an important question arose.

Problem. For CNN with/without input, if the radius of the interacting cells $d$ is treated as a parameter, is $\{h(A, B, z, d)\} /\{h(A, z, d)\}$ dense in $[0, \log 2]$ ?

Multifractal analysis is introduced to a specified dynamical system when one of its invariants is essentially the same as an interval (see [Pesin, 1997] for more detail), this motivates us to consider such a problem. However, since the wellknown fact that the entropy of subshift of finite type takes a family of specific values, called Perron number [Lind \& Marcus, 1995], the "dense" assumption cannot be removed. The main difficulty in solving the problem is related to the fact that the admissible local patterns that are produced by CNN are very limited [Hsu et al., 2000; Juang \& Lin, 2000]. Restated, there exists $\mathcal{U} \subseteq$ $\{1,-1\}^{\mathbb{Z}_{n \times n}}$ such that $\mathcal{U} \neq \mathcal{B}(A, z, d) / \mathcal{B}(A, B, z, \bar{d})$ for all chosen values of the parameters $A, B, z, d$, where $n=2 d+1$.

For example, consider the one-dimensional CNN without input, and the length of interaction $d=1$. Figure 1 is the bifurcation diagram that relates admissible local patterns to the parameters $A=\left(a_{l}, a, a_{r}\right)$ and $z$; readers may refer to [Hsu et al., 2000; Juang \& Lin, 2000] for

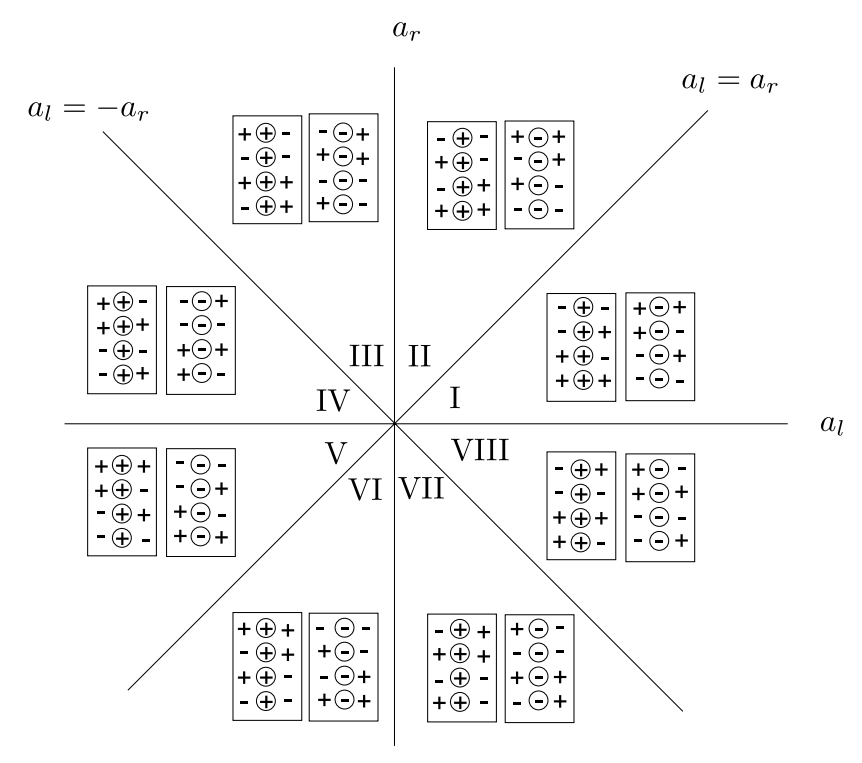

Fig. 1. The bifurcation diagram of 1-D CNN.

more details. First, choosing $\left(a_{l}, a_{r}\right)$ yields a total of eight partitions, as shown in Fig. 1. Second, the $(a-1, z)$ plane has 25 regions such that the admissible local patterns will be uniquely determined once the region is chosen. For instance, if the parameters $A, z$ are in region $[3,4]$ of partition IV, the admissible local patterns are

$$
\begin{aligned}
\mathcal{B}= & \{-\oplus+,-\oplus-,+\oplus+,+\ominus-,+\ominus+, \\
& -\ominus-,-\ominus+\} .
\end{aligned}
$$

That is, "3" indicates that the three patterns with "+" in the center should be chosen from the bottom, and "4" indicates that all four patterns with "_" in the center can be chosen in IV. Thus, Figs. 1 and 2 show all admissible local patterns of 1-D CNN with $d=1$.

However, let $\mathcal{U} \subseteq\{1,-1\}^{\mathbb{Z}_{3 \times 1}}$ be the set of patterns which are listed as follows.

$\mathcal{U}=\{-\oplus-,-\oplus+,+\oplus-,-\ominus-,-\ominus+,+\ominus-\}$.

Notably, $\mathcal{U}$ consists of patterns that are selected from different partitions for $a_{l}$ and $a_{r}$. More precisely, the patterns with "+" in the center are located in partition $\mathrm{V}$ such that the parameters $a_{l}$ and $a_{r}$ must satisfy the conditions $a_{l}<0$ and $a_{r}>0$. Moreover, the patterns with "_" in the center are selected from partition I, in which the associated parameters $a_{l}, a_{r}$ must then satisfy $a_{l}, a_{r}>0$. Accordingly, there does not exist $A, z$ such that $\mathcal{B}(A, z)=\mathcal{U}$. Thus, some values of entropy cannot be attained for all choices of $3 \times 1$ basic sets for $d=1$. 
This study elucidates the ICNN system (defined later). Most materials in natural systems, including physical, biological and electronic systems, are spatially inhomogeneous [Ferdinand \& Fisher, 1969; Perdew, 1986; Rosenfeld, 1989; Kravtsov \& Orlov, 1990; Pesin, 1997; Debye \& Bueche, 2004]. The ICNN system is of the form,

$$
\frac{d x_{i, j}}{d t}= \begin{cases}-x_{i, j}+z+\sum_{|k|,|l| \leq d} a_{k, l} f\left(x_{i+k, j+l}\right)+\sum_{|k|,|l| \leq d} b_{k, l} u_{i+k, j+l}, & i, j \equiv 0 \bmod m \\ -x_{i, j}+z^{\prime}+a_{0,0} f\left(x_{i, j}\right), & \text { otherwise }\end{cases}
$$

for some $m \in \mathbb{N}, i, j \in \mathbb{Z}$. Restated, the difference between CNN and ICNN is that the templates and threshold at each cell $C_{i, j}$ are spatially invariant for CNN but variant for ICNN. This work presents a solution to the problem of ICNN, but does not solve the problem of CNN. The authors suspect the answer to the problem of $\mathrm{CNN}$ is also positive.

In a work on dense entropy, Quas and Trow [2000] showed that every subshift of finite type (SFT) $\mathbf{X}$ with positive entropy has proper SFT $\mathbf{X}^{\prime}$ which is a subsystem of $\mathbf{X}$ whose entropy is strictly less than the entropy of $\mathbf{X}$, but whose entropy is arbitrarily close to that of $\mathbf{X}$. However, they cannot be guaranteed to be mixing [Quas \& Sahin, 2003]. Recently, Desai [2006] proved that for any $\mathbb{Z}^{d}$-SFT $\mathbf{R}$ of positive entropy, the SFT subsystems achieve dense entropy in $[0, h(\mathbf{R})]$. Thus, if $\mathbf{R}$ is treated as a full shift, then the SFT is dense in $[0, \log |\mathcal{A}|]$, where $\mathcal{A}$ denotes the symbols of $\mathbf{R}$, and this result can be generalized to sofic systems. Restated, given

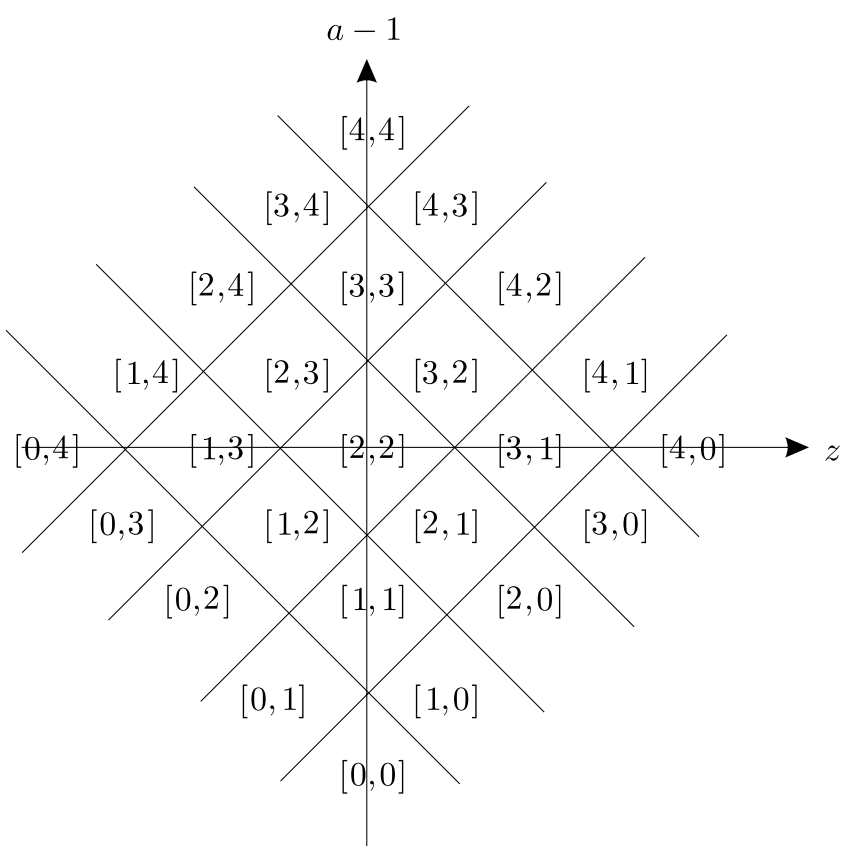

Fig. 2. The partition of $a-z$ plane of $1-\mathrm{D}$ CNN. a $\mathbb{Z}^{d}$ sofic shift $\mathbf{T}$, the sofic shift subsystems achieve dense entropy in $[0, h(\mathbf{T})]$. However, a difficulty similar to that associated with CNN arises in solving the problem of ICNN. The difficulty is to guarantee that the patterns that would achieve the desired entropy can be produced by an ICNN system with/without input. This investigation proposes a necessary and sufficient condition for the admissibility of local patterns of ICNN, and demonstrates that suitable local patterns can be found that achieve the given $t \in[0, \log 2]$ (according to Theorem A for ICNN without input and Theorem $\mathrm{B}$ for the case with input). Finding these patterns solves the dense entropy problem for ICNN.

The rest of this paper is organized as follows. Section 2 introduces preliminaries that constitute the background for this work. Section 3 then presents a general theory that yields details about how ICNN relates to a shift of finite type. The solution to the dense entropy problem is also addressed. Section 4 extends the results in Sec. 3 to ICNN with input.

\section{Preliminary}

Several notions for the formulation of the main results in Secs. 3 and 4 are presented in this section. Since the states $C_{i, j}$ with $i=k_{1} m, j=k_{2} m$ for $k_{1}, k_{2} \in \mathbb{Z}$ are crucial for the study of the mosaic solutions of ICNN, these cells are the main focus in the rest of this investigation.

Definition 2.1. Let $\bar{x}=\left(\bar{x}_{i, j}\right)$ be the stationary solution of system (2). $\bar{x}$ is called a mosaic solution if $\left|\bar{x}_{i, j}\right|>1$ for all $i, j \in \mathbb{Z}$, and is called an interior solution if $\left|\bar{x}_{i, j}\right|<1$ for all $i, j \in \mathbb{Z}$. A defect solution $\bar{x}$ satisfies $\left|\bar{x}_{i, j}\right|>1$ for some $(i, j) \in D$ and $\left|\bar{x}_{k, \ell}\right|<1$ for some $(k, \ell) \notin D$, where $D \subsetneq \mathbb{Z}^{2}$ and $D \neq \varnothing$.

First, considering the system (2) without input, that is, the template $B \equiv 0$. For each given mosaic solution $\bar{x}$, the output pattern at cell $C_{i, j}$ is + , i.e. 
$\bar{x}_{i, j}>1$, if and only if

$$
\sum_{\substack{|k|,|l| \leq d \\(k, l) \neq(0,0)}} a_{k, l} \bar{y}_{i+k, j+l}+a+z-1>0,
$$

where $a_{0,0} \equiv a$. Similarly, the output pattern at cell $C_{i, j}$ is - , i.e. $\bar{x}_{i, j}<-1$, if and only if

$$
\sum_{\substack{|k|,|l| \leq d \\(k, l) \neq(0,0)}} a_{k, l} \bar{y}_{i+k, j+l}-a+z+1<0 .
$$

(3) and (4) can be rewritten in a much more compact form by introducing the following notations.

Denote $n=4 d^{2}+4 d$. Let $X^{n}$ be the $n$ dimensional lattice points, i.e.

$X^{n}=\left\{v=\left(v_{i}\right) \in \mathbb{R}^{n}:\left|v_{i}\right|=1\right.$ for $\left.1 \leq i \leq n\right\}$.

Then, for a given pair of template $A$ and threshold $z$, the basic set of admissible local patterns with "+" state in the center is defined by

$$
\mathcal{B}(+, A, z, d)=\left\{v \in X^{n}: \alpha \cdot v+a+z-1>0\right\}
$$

where "." is the inner product, $\alpha=\left(a_{1}, a_{2}, \ldots, a_{n}\right), v=\left(v_{1}, v_{2}, \ldots, v_{n}\right)$ are obtained from

$$
\left[\begin{array}{ccccc}
a_{4 d^{2}+2 d} & \cdots & a_{2 d^{2}+d+1} & \cdots & a_{1} \\
& & \vdots & & \\
\vdots & \cdots & a_{i, j} & \cdots & \vdots \\
& & \vdots & & \\
a_{n} & \cdots & a_{2 d^{2}+3 d} & \cdots & a_{2 d+1}
\end{array}\right]=\left[\begin{array}{ccccc}
a_{i-d, j+d} & \cdots & a_{i, j+d} & \cdots & a_{i+d, j+d} \\
& & \vdots & & \\
\vdots & \cdots & a_{i, j} & \cdots & \vdots \\
& & \vdots & & \\
& & & & \\
a_{i-d, j-d} & \cdots & a_{i, j-d} & \cdots & a_{i+d, j-d}
\end{array}\right]
$$

and

$$
\left[\begin{array}{ccccc}
v_{4 d^{2}+2 d} & \cdots & v_{2 d^{2}+d+1} & \cdots & v_{1} \\
& & \vdots & & \\
\vdots & \cdots & v_{i, j} & \cdots & \vdots \\
& & \vdots & & \\
v_{n} & \cdots & v_{2 d^{2}+3 d} & \cdots & v_{2 d+1}
\end{array}\right]=\left[\begin{array}{ccccc}
y_{i-d, j+d} & \cdots & y_{i, j+d} & \cdots & y_{i+d, j+d} \\
& & \vdots & & \\
\vdots & \cdots & y_{i, j} & \cdots & \vdots \\
& & \vdots & & \\
y_{i-d, j-d} & \cdots & y_{i, j-d} & \cdots & y_{i+d, j-d}
\end{array}\right],
$$

respectively. In other words, $\alpha$ represents the surrounding template of $A$ without center, and $v$ indicates the output patterns at cell $C_{i, j}$ whose center is omitted. Similarly, the basic set of admissible local patterns with "-" in the center is defined by

$$
\mathcal{B}(-, A, z, d)=\left\{v \in X^{n}: \alpha \cdot v-a+z+1<0\right\} .
$$

An investigation of the basic sets of admissible local patterns $\mathcal{B}(+, A, z, d)$ and $\mathcal{B}(-, A, z, d)$ is essential for the understanding of the global mosaic patterns on $\mathbb{Z}^{2}$ that are generated by the given $(A, z)$. Some definitions and theorems should be stated first.

Definition 2.2. Given $\mathcal{U} \subset X^{n}, \mathcal{U}$ is called separable if there is a hyperplane $H$ in $\mathbb{R}^{n}$ such that $\mathcal{U}$ and $\mathcal{U}^{c}$ can be separated by $H$, where $\mathcal{U}^{c}=X^{n} \backslash \mathcal{U}$.

Hsu et al. [2000] investigated how the admissible local mosaic patterns $\mathcal{B}(*, A, z, d)$ relate to the parameters $A, z$ and $d$ in CNN systems, where $* \in\{+,-\}$.

Theorem 2.3 [Hsu et al., 2000]. There exists $(A, z)$ and $d$ such that $\mathcal{U}=\mathcal{B}(*, A, z, d)$ for some $* \in$ $\{+,-\}$ if and only if $\mathcal{U}$ is separable.

Moreover, the classical theory of convex set [Lay, 1992] gives the necessary and sufficient condition when $\mathcal{U} \subseteq X^{n}$ is separable.

Theorem 2.4 (Linear Separating Theorem). $\mathcal{U}$ and $\mathcal{U}^{c}$ can be separated by a hyperplane in $\mathbb{R}^{n}$ if and only if

$$
\operatorname{conv}(\mathcal{U}) \cap \operatorname{conv}\left(\mathcal{U}^{c}\right)=\varnothing,
$$

where $\operatorname{conv}(\mathcal{K})$ is the convex hull of $\mathcal{K}$ in $\mathbb{R}^{n}$.

Let $\mathbf{z}=\left(z, z^{\prime}\right)$ denote the thresholds, and let $\mathcal{B}(A, \mathbf{z}) / \mathcal{B}(A, B, \mathbf{z})$ denote the basic set of admissible local patterns of ICNN without/with input for 
the given templates. The result in Theorem 2.3 still holds for ICNN systems.

Theorem 2.5. There exists $(A, \mathbf{z})$ and $d$ such that $\mathcal{U}=\mathcal{B}(*, A, \mathbf{z}, d)$ for some $* \in\{+,-\}$ if and only if $\mathcal{U} \subseteq X^{n}$ is separable.

Proof. It suffices to show that $\mathcal{B}(+, A, \mathbf{z}, d)=\mathcal{U}$ for some $(A, \mathbf{z})$ and $d$ if and only if $\mathcal{U}$ is separable. The proof for $*=-$ is essentially the same, thus is omitted.

First, considering the output pattern at $C_{i, j}$, where $(i, j) \neq\left(k_{1} m, k_{2} m\right)$ for some $k_{1}, k_{2} \in \mathbb{Z}$. The output pattern is + if and only if $a+z^{\prime}-1>0$, and is - if and only if $a-z^{\prime}-1>0$. Let $a>1$ and $z^{\prime}=1 / 2(a-1)$. The output pattern at $C_{i, j}$ can be arbitrary in such a case. It remains to show that $\mathcal{U}$ can be realized on $C_{i, j}$ for some appropriate choice of $(A, z)$, where $i, j \equiv 0 \bmod m$.

Let $S=\left\{\mathcal{U} \subseteq X^{n} \mid \mathcal{U}\right.$ satisfies (6) $\}$. For each $\mathcal{U} \in S$, denoted by

$\mathcal{A}^{+}(\mathcal{U})=\{(\alpha, p) \mid \alpha \cdot v+p>0$ for all $v \in \mathcal{U}\}$,

$\mathcal{A}^{-}(\mathcal{U})=\left\{(\alpha, q) \mid \alpha \cdot v+q<0\right.$ for all $\left.v \in \mathcal{U}^{c}\right\}$.

Then $\mathcal{A}^{+}(\mathcal{U}) \cap \mathcal{A}^{-}(\mathcal{U}) \neq \varnothing$ if and only if $\mathcal{U}$ satisfies (6). In this case, the boundary $\partial \mathcal{A}^{+}(\mathcal{U})$ of $\mathcal{A}^{+}(\mathcal{U})$ consists of $(A, B, z)$ such that $\alpha \cdot v+a+z-1=0$, where $p=a+z-1$.

Defining

$$
\hat{\mathcal{B}}(+, \alpha, p)=\{v: \alpha \cdot v+p>0\},
$$

then $\hat{\mathcal{B}}(+, \alpha, p)=\mathcal{U}$ for all $(\alpha, p) \in \mathcal{A}^{+}(\mathcal{U})$. For each $\mathcal{U} \in S$ so that there exists $(\alpha, p) \in \mathcal{A}^{+}(\mathcal{U})$,

consider

$$
z=\frac{p}{2}-k, \quad a=1+\frac{p}{2}+k,
$$

where $k$ is chosen so that $p / 2+k>0$. Then $\mathcal{B}(+, A, \mathbf{z}, d)=\hat{\mathcal{B}}(+, \alpha, p)=\mathcal{U}$, and vice versa. This completes the proof.

Next, considering system (2) with input. Given a mosaic solution $\bar{x}$, the output pattern at cell $C_{i, j}$ is + if and only if

$$
\begin{aligned}
& \sum_{\substack{|k|,|l| \leq d \\
(k, l) \neq(0,0)}} a_{k, l} \bar{y}_{i+k, j+l}+\sum_{|k|,|l| \leq d} b_{k, l} \bar{u}_{i+k, j+l} \\
& \quad+a+z-1>0 .
\end{aligned}
$$

Similarly, the output pattern at cell $C_{i, j}$ is - if and only if

$$
\begin{aligned}
& \sum_{\substack{|k|,|l| \leq d \\
(k, l) \neq(0,0)}} a_{k, l} \bar{y}_{i+k, j+l}+\sum_{|k|,|l| \leq d} b_{k, l} \bar{u}_{i+k, j+l} \\
& \quad-a+z+1<0 .
\end{aligned}
$$

It is seen from the above discussion that the basic set of admissible local patterns with "+" in the center is defined by

$$
\begin{aligned}
\mathcal{B}(+, A, B, z, d)= & \left\{(v, w) \in X^{n} \times X^{n+1}: \alpha \cdot v\right. \\
& +\beta \cdot w+a+z-1>0\},
\end{aligned}
$$

and the basic set of admissible local patterns with "_" in the center is defined by

$$
\begin{aligned}
\mathcal{B}(-, A, B, z, d)= & \left\{(v, w) \in X^{n} \times X^{n+1}: \alpha \cdot v\right. \\
& +\beta \cdot w-a+z+1<0\} .
\end{aligned}
$$

Herein, $\beta=\left(b_{1}, b_{2}, \ldots, b_{n+1}\right)$ and $w=\left(w_{1}\right.$, $\left.w_{2}, \ldots, w_{n+1}\right)$ are obtained from

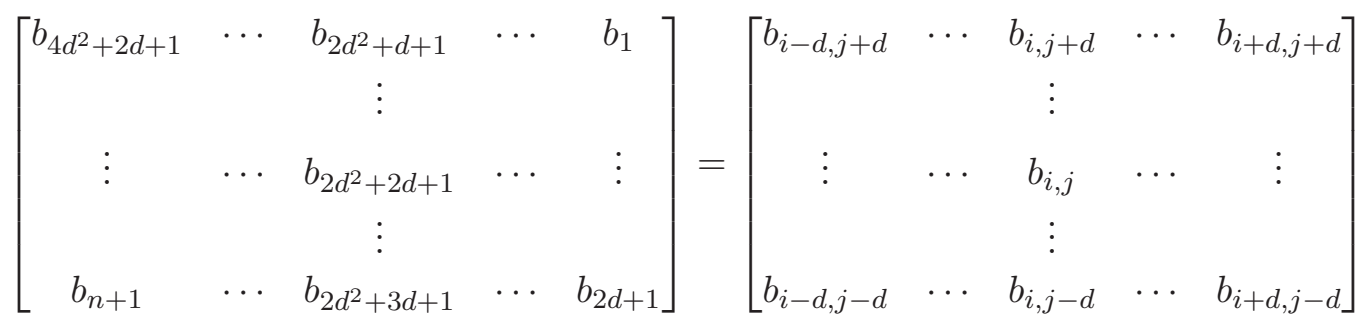

and

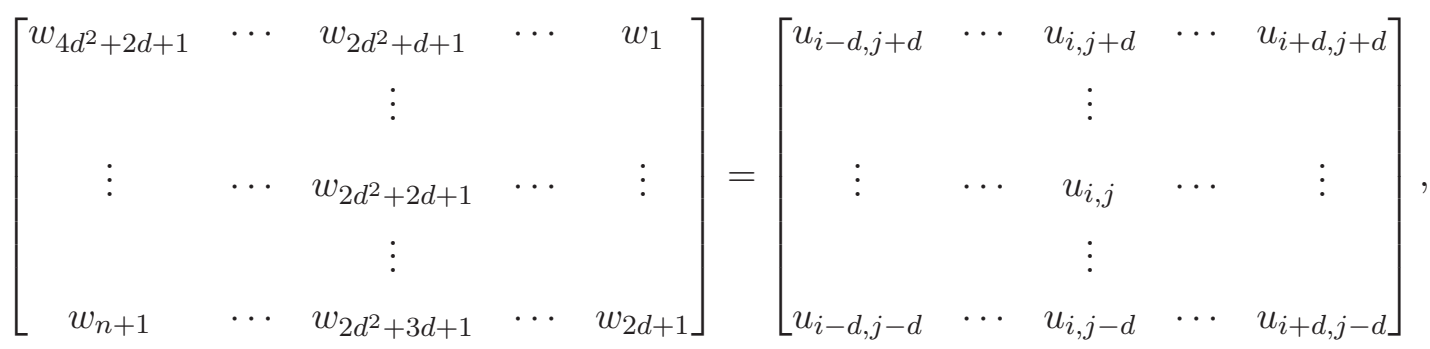


respectively. Namely, $\beta$ represents the template $B$ and $w$ indicates the input patterns at cell $C_{i, j}$.

Ban et al. [2007b] generalized Theorem 2.3 to a common case that the controlling template $B$ is considered.

Theorem 2.6 [Ban et al., 2007b]. There exists $(A, B, z)$ and $d$ such that $\mathcal{U}=\mathcal{B}(*, A, B, z, d)$ for some $* \in\{+,-\}$ if and only if $\mathcal{U}$ is separable.

Theorem 2.6 can also be applied for ICNN with input.

Theorem 2.7. There exists $(A, B, \mathbf{z})$ and $d$ such that $\mathcal{U}=\mathcal{B}(*, A, B, \mathbf{z}, d)$ for some $* \in\{+,-\}$ if and only if $\mathcal{U}$ is separable.

Proof. This can be accomplished via analogous method as in the proof of Theorem 2.5, thus is omitted.

\section{Inhomogeneous Cellular Neural Networks without Input}

The dense entropy property for the ICNN without input is studied in this section. Section 3.1 develops the fundamental theory and presents its application for ICNN in Sec. 3.2.

\subsection{Two-dimensional subshift of finite type}

This subsection investigates the preliminaries that are necessary for the understanding of dense entropy property of ICNN without input.

Definition 3.1. Let $\mathbf{X} \subseteq\{1,-1\}^{\mathbb{Z}^{2}}$ be a twodimensional shift space with finite alphabet $\mathcal{A}(\mathbf{X})=\{1,-1\}$.

(1) If $x \in \mathbf{X}$ and $S \subseteq \mathbb{Z}^{2}$, the restriction of $x$ to $S$ is denoted by $\pi_{S}(x)$.

(2) Let $\Lambda(n)=\{(p, q): p, q \in \mathbb{Z}, 0 \leq p, q \leq n-1\}$. An $n$-block is $\pi_{\mathbf{c}+\Lambda(n)}(x)$ for some $\mathbf{c} \in \mathbb{Z}^{2}$, $x \in \mathbf{X}$. The set of $n$-blocks is denoted by $B_{n}(\mathbf{X})$.

(3) A configuration on $S \subseteq \mathbb{Z}^{2}$ is a map $E: S \rightarrow$ $\mathcal{A}(\mathbf{X})$. For $x \in \mathbf{X}, E$ occurs in $x$ if $\pi_{\mathbf{c}+S}(x)=E$ for some $\mathbf{c} \in \mathbb{Z}^{2}$.

(4) For each $\mathbf{c} \in \mathbb{Z}^{2}$, the shift map $\sigma_{\mathbf{c}}: \mathbf{X} \rightarrow \mathbf{X}$ is defined by $\pi_{\mathbf{d}}\left(\sigma_{\mathbf{c}}(x)\right)=\pi_{\mathbf{c}+\mathbf{d}}(x)$ for all $\mathbf{d} \in \mathbb{Z}^{2}$. Moreover, the iteration of $\sigma_{\mathbf{c}}$ is denoted by $\sigma_{\mathbf{c}}^{\ell}=\sigma_{\mathbf{c}} \circ \sigma_{\mathbf{c}}^{\ell-1}$ for all $\ell \in \mathbb{N}$.

Denote $\pi_{\Lambda(n)}(x)$ by $\pi_{n}(x)$ for simplicity.
Definition 3.2. Given $\mathcal{U} \subseteq\{1,-1\}^{\mathbb{Z}_{n \times n}}, s \in \mathbb{N}, s<$ $n$, the shift space $\mathbf{X}_{s}(\mathcal{U}) \subseteq\{1,-1\}^{\mathbb{Z}^{2}}$ is defined by

$$
\begin{aligned}
\mathbf{X}_{s}(\mathcal{U})= & \left\{x \in\{1,-1\}^{\mathbb{Z}^{2}}: \pi_{n}\left(\sigma_{(i, j)}^{\ell}(x)\right) \in \mathcal{U}\right. \\
& \text { for all } \ell \in \mathbb{Z}, i, j \in\{0, n-s\}\} .
\end{aligned}
$$

Moreover, the $r$-copy of $\mathcal{U}, \mathcal{U}^{r} \subseteq\{1,-1\}^{\mathbb{Z}_{k \times k}}$, where $k=r n-(r-1) s$, is defined by

$$
\begin{aligned}
\mathcal{U}^{r}= & \left\{v \in\{1,-1\}^{\mathbb{Z}_{k \times k}}: \exists x \in \mathbf{X}_{s}(\mathcal{U})\right. \\
& \text { such that } \left.\pi_{k}(x)=v\right\} .
\end{aligned}
$$

Remark 3.3. In other words, $\mathcal{U}^{r}$ consists of those patterns combined by $r^{2}$-many patterns in $\mathcal{U}$ with $s$-many rows/columns overlapped. For example, consider $\mathcal{U} \subseteq\{1,-1\}^{\mathbb{Z}_{4 \times 4}}$ and $s=1 . \mathcal{U}^{2}$ consists of those patterns with size $7 \times 7$ such that each pattern $v \in \mathcal{U}^{2}$ is a combination of four patterns in $\mathcal{U}$ with one-row/column overlapped. As seen in Fig. 3, the last column on the right-hand side in pattern 1 can be overlapped with the first column on the lefthand side in pattern 2 if and only if these two $1 \times 4$ patterns are exactly the same. The same applies to the top row in pattern 1 and the bottom row in pattern 3 .

Next, the effect of the parameter $s$ is studied. In general, the range of $s$ is less than $n$ and greater than one. After constructing $\mathcal{U}^{r}$ from a given $\mathcal{U}$, the lemma below studies the relationship between the subshifts of finite types $\mathbf{X}_{s}(\mathcal{U})$ and $\mathbf{X}_{s}\left(\mathcal{U}^{r}\right)$. In addition, it reduces the complexity caused by $s$.

Lemma 3.4. Given $\mathcal{U} \subseteq\{1,-1\}^{\mathbb{Z}_{n \times n}}$ and $r \in \mathbb{N}$, then $\mathbf{X}_{s}(\mathcal{U})=\mathbf{X}_{s}\left(\mathcal{U}^{r}\right)$.

Proof. Since $\mathcal{U}^{r}$ is constructed from $\mathcal{U}$ such that each pattern in $\mathcal{U}^{r}$ consists of $r^{2}$-many patterns in $\mathcal{U}$ with $s$-many columns/rows overlapped, it is seen that $\mathbf{X}_{s}\left(\mathcal{U}^{r}\right) \subseteq \mathbf{X}_{s}(\mathcal{U})$. This remains to show that $\mathbf{X}_{s}(\mathcal{U}) \subseteq \mathbf{X}_{s}\left(\overline{\mathcal{U}}^{r}\right)$.

If $x \in \mathbf{X}_{s}(\mathcal{U})$, then $\pi_{n}\left(\sigma_{(i, j)}^{\ell}(x)\right) \in \mathcal{U}$ for all $\ell \in \mathbb{Z}$, where $i, j \in\{0, n-s\}$. Definition 3.2 shows that $\pi_{k}(x) \in \mathcal{U}^{r}$, where $k=r n-(r-1) s-1$. Let $y=\sigma_{(i, j)}(x)$ for some $i, j \in\{0, n-s\}$. Then $\pi_{k}(y) \in \mathcal{U}^{r}$ via the same argument. It can be easily checked that $\pi_{k}\left(\sigma_{(i, j)}^{\ell}(x)\right) \in \mathcal{U}$ for all $\ell \in \mathbb{Z}$ by mathematical induction, where $i, j \in\{0, n-s\}$. Therefore, $x \in \mathbf{X}_{s}\left(\mathcal{U}^{r}\right)$ and this completes the proof.

Without loss of generality, assuming that $s \leq$ $[n / 2]$, where [.] is the Gauss function. The case where $s>[n / 2]$ is discussed in Remark 3.8. 


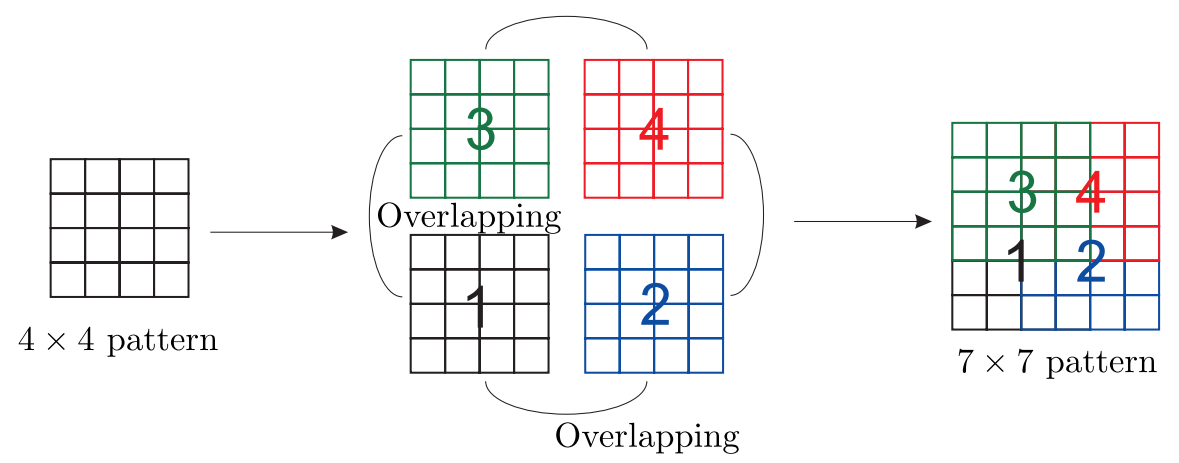

(a)
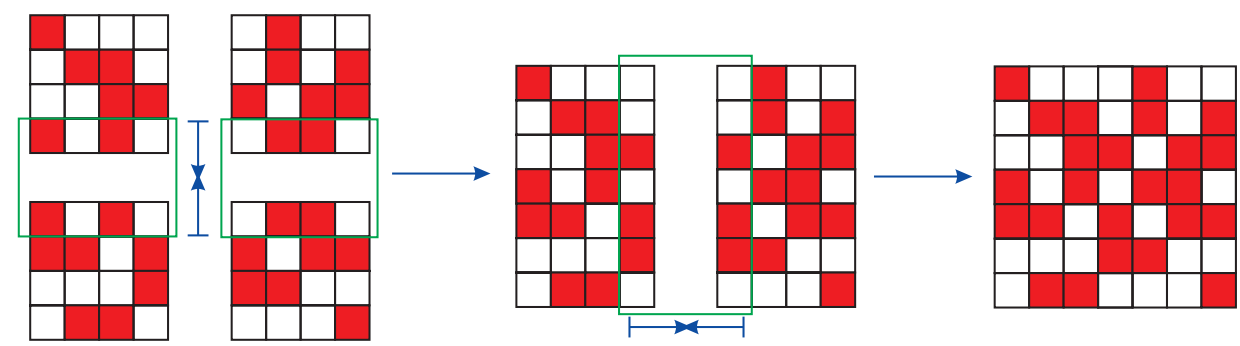

(b)

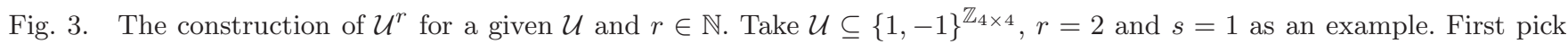
four patterns in $\mathcal{U}$, say $P_{1}, P_{2}, P_{3}, P_{4}$. If the patterns in the first row of $P_{1}$ differ from the patterns in the last row of $P_{3}$, then nothing happens. Otherwise, $P_{1}$ and $P_{3}$ are combined with one-row overlapped. Repeating this process, a new pattern with size $7 \times 7$ is thus derived.

It is seen so far that a subshift of finite type is generated once $\mathcal{U}$ and $s$ are given. The method that embeds a chosen set of admissible local patterns in an ICNN system is introduced.

If $\mathcal{U} \subseteq\{1,-1\}^{\mathbb{Z}_{n \times n}}$ is given and $n$ is even, then an extension of $\mathcal{U}$, denoted by $\mathcal{V} \subset\{1,-1\}^{\mathbb{Z}_{(n+1) \times(n+1)}}$, is constructed as follows. $v=\left(v_{(i, j)}\right) \in \mathcal{V}$ if and only if

(i) $v_{(i, j)}=-1$ if $i=(n / 2)+1$ or $j=(n / 2)+1$.

(ii) $v_{\langle(n / 2)+1\rangle}=u$ for some $u \in \mathcal{U}$, where $v_{\langle p ; q\rangle} \in$ $\{1,-1\}^{\mathbb{Z}_{n \times n}}$ is obtained from $v$ by deleting row $p$ and column $q$, and denoted by $v_{\langle p\rangle}$ if $p=q$.

Similarly, if $n$ is odd, constructing $\mathcal{V} \subset$ $\{1,-1\}^{\mathbb{Z}_{(n+2) \times(n+2)}}$ by $v=\left(v_{(i, j)}\right) \in \mathcal{V}$ if and only if

(i) $v_{(i, j)}=-1$ if either $i$ or $j \in\{(n+1) / 2$, $(n+3) / 2\}$.

(ii) $v_{\langle(n-1) / 2\rangle}^{\prime}=u$ for some $u \in \mathcal{U}$, where $v_{\langle p ; q\rangle}^{\prime} \in$ $\{1,-1\}^{\mathbb{Z}_{n \times n}}$ is obtained from $v_{\langle p ; q\rangle}$ by deleting row $p$ and column $q$, and denoted by $v_{\langle p\rangle}^{\prime}$ if $p=q$.
More precisely, $\mathcal{U}$ is extended to $\mathcal{V}$ by adding a cross of " -1 " to the center of each $u \in \mathcal{U}$. Under such extension, there is a one-to-one correspondence between $\mathcal{U}$ and $\mathcal{V}$. Figure 4 gives two examples for the cases where $n$ is odd and $n$ is even, respectively.

Remark 3.5. Notably, the size of $\mathcal{V}$ is odd no matter what the size of $\mathcal{U}$ is. That is, $\mathcal{V} \subseteq\{1,-1\}^{\mathbb{Z}_{\ell \times \ell}}$ for some $\ell=2 k+1, k \in \mathbb{N}$.

For each $\mathcal{U} \subseteq\{1,-1\}^{\mathbb{Z}_{n \times n}}$, there associates an unique $\mathcal{V} \subseteq\{1,-1\}^{\mathbb{Z}_{(n+1) \times(n+1)}}$ under the construction above. The relationship between $\mathbf{X}_{s}(\mathcal{U})$ and $\mathbf{X}_{s}(\mathcal{V})$ is investigated below. Before stating the lemma, a definition is given first.

Definition 3.6. Let $\mathbf{X}, \mathbf{Y}$ be shift spaces with shift maps $\sigma_{\mathbf{X}}$ and $\sigma_{\mathbf{Y}}$, respectively. Define $\phi: \mathbf{X} \rightarrow \mathbf{Y}$ be a factor map from $\mathbf{X}$ to $\mathbf{Y}$ if $\phi$ is onto and $\phi \circ \sigma_{\mathbf{X}}=\sigma_{\mathbf{Y}} \circ \phi . \mathbf{X}$ is conjugate to $\mathbf{Y}$, denoted by $\mathbf{X} \cong \mathbf{Y}$, if $\phi$ is a factor map and one-to-one.

A key lemma then follows.

Lemma 3.7. Given $\mathcal{U} \subseteq\{1,-1\}^{\mathbb{Z}_{n \times n}}$, constructing $\mathcal{V}$ as above, then $\mathbf{X}_{s}(\mathcal{U}) \cong \mathbf{X}_{s}(\mathcal{V})$. 

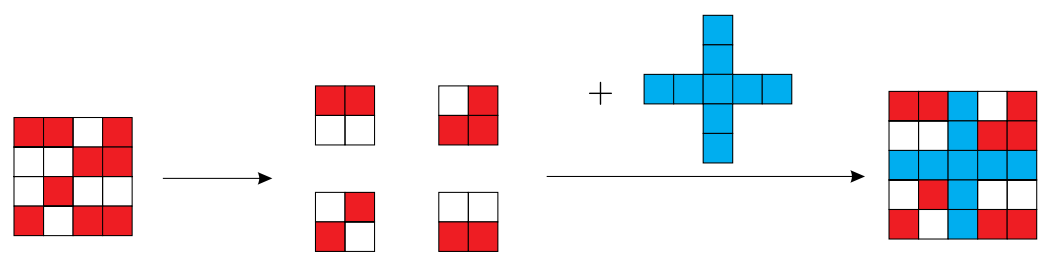

(a)

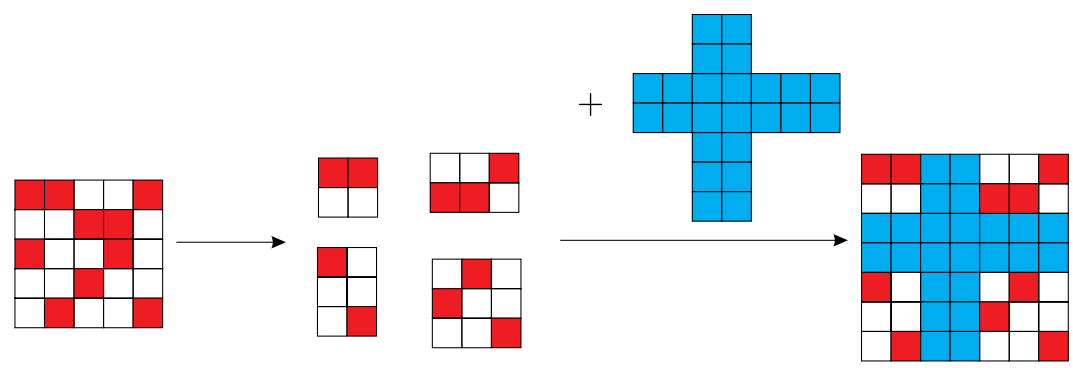

(b)

Fig. 4. (a) Extend a $4 \times 4$ pattern to a $5 \times 5$ pattern by adding a cross of pattern into the center of the original one. The pattern "+" is represented by red and the pattern "-" is represented by white and blue, herein blue is used to distinguish from the original pattern. (b) Extend a $5 \times 5$ pattern to a $7 \times 7$ pattern.

Proof. Define $\psi: \mathcal{V} \rightarrow \mathcal{U}$ by $\psi(v)=u$, where

$$
u= \begin{cases}v_{\left\langle\frac{n}{2}+1\right\rangle}, & n \text { is even; } \\ v_{\left\langle\frac{n-1}{2}\right\rangle}^{\prime}, & n \text { is odd. }\end{cases}
$$

For simplicity, assume $n$ is even. The case where $n$ is odd can be done similarly. It is easily seen that $\psi(v)$ is bijective. Furthermore, defining $\phi: \mathbf{X}_{s}(\mathcal{V}) \rightarrow$ $\mathbf{X}_{s}(\mathcal{U})$ by $\left.\phi(y)_{(\ell i, \ell j)+\mathbf{c}}=\psi\left(\pi_{n+1}\left(y_{(\ell q i, \ell q j}\right)\right)\right)_{\mathbf{c}}$, where $i, j \in\{0, n-s\}, \ell \in \mathbb{Z}, q=(n-s+1) /(n-s), \mathbf{c} \in$ $\Lambda(n)$ and $y \in \mathbf{X}_{s}(\mathcal{V})$. In such a case, $\phi \circ \sigma_{\mathbf{X}_{s}(\mathcal{V})}=$ $\sigma_{\mathbf{X}_{s}(\mathcal{U})} \circ \phi$ and $\phi$ is a conjugacy since $\psi$ is one-to-one and onto. This completes the proof.

Remark 3.8. If $s>[n / 2]$, let $\ell \in \mathbb{N}$ satisfy

$$
\left[\frac{(\ell-1)(n-s)+s}{2}\right]<s \leq\left[\frac{\ell(n-s)+s}{2}\right] \text {. }
$$

Then construct $\mathcal{V}$ via the same method mentioned above so that there is a one-to-one correspondence between $\mathcal{V}$ and $\mathcal{U}^{\ell}$. Similar as above, Lemmas 3.4 and 3.7 show that $\mathbf{X}_{s}(\mathcal{U}) \cong \mathbf{X}_{s}(\mathcal{V})$.

\subsection{Two-dimensional inhomogeneous cellular neural networks without input}

Section 3.1 shows that $\mathbf{X}_{s}(\mathcal{U})=\mathbf{X}_{s}\left(\mathcal{U}^{r}\right)$ and $\mathbf{X}_{s}(\mathcal{U}) \cong \mathbf{X}_{s}(\mathcal{V})$, where $\mathcal{U} \subseteq\{1,-1\}^{\mathbb{Z}_{n \times n}}$ is given, $\mathcal{V} \subseteq\{1,-1\}^{\mathbb{Z}_{(n+1) \times(n+1)}}$ is obtained from $\mathcal{U}$ and $r \in \mathbb{N}$. This subsection applies the theory developed in the last subsection to ICNN without input. First, the preservation of the separation property between $\mathcal{U}$ and $\mathcal{V}$ is given below.

Lemma 3.9. Given $\mathcal{U} \subseteq\{1,-1\}^{\mathbb{Z}_{n \times n}}$, then $\mathcal{U}$ is separable if and only if $\mathcal{V}$ is separable.

Proof. For simplicity, the case where $n$ is even is proved. It can be done similarly when $n$ is odd.

If $\mathcal{U}$ is separable, there is a linear functional $g:\{1,-1\}^{\mathbb{Z}_{n \times n}} \rightarrow \mathbb{R}$ and $\alpha \in \mathbb{R}$ so that $g(u)<\alpha$ for all $u \in \mathcal{U}$, and $g(u)>\alpha$ for all $u \in \mathcal{U}^{c}$. Let $\rho=\alpha-\min \{g(u): u \in \mathcal{U}\}$. Define $\hat{g}$ : $\{1,-1\}^{\mathbb{Z}_{(n+1) \times(n+1)}} \rightarrow \mathbb{R}$ by

$$
\hat{g}(v)=g(u)+\rho \sum_{i \text { or }} \sum_{=(n / 2)+1} v_{(i, j)},
$$

where $u \in\{1,-1\}^{\mathbb{Z}_{n \times n}}$ is obtained from $v$ by deleting row $((n / 2)+1)$ and column $((n / 2)+1)$. Then $\hat{g}(v)<\alpha-(2 n+1) \rho$ for $v \in \mathcal{V}$ and $\hat{g}(v)>$ $\alpha-(2 n+1) \rho$ for $v \in \mathcal{V}^{c}$. Thus, $\mathcal{V}$ is separable.

Similarly, if $\mathcal{V}$ is separable, then so is $\mathcal{U}$. This completes the proof.

Before stating the main theorem, the following theorem is essential for the study of the mosaic solutions of ICNN. 
Theorem 3.10. Given $\mathcal{U} \subseteq\{1,-1\}^{\mathbb{Z}_{n \times n}}$, and $s \in \mathbb{N}$. If $\mathcal{U}$ is separable, then there exist $m \in \mathbb{N}$ and $(A, \mathbf{z}, d)$ for system (2) such that $\mathbf{X}(\mathcal{B}(A, \mathbf{z}, d)) \cong \mathbf{X}_{s}(\mathcal{U})$, where $\mathcal{B}(A, \mathbf{z}, d)$ is the admissible local patterns obtained from (2) with parameters $(A, \mathbf{z}, d)$,

$$
\mathbf{X}(\mathcal{B}(A, \mathbf{z}, d))=\left\{\begin{array}{c}
x \in\{1,-1\}^{\mathbb{Z}^{2}}: \pi_{\kappa(d)}\left(\sigma_{(i, j)}^{\ell}(x)\right) \in \mathcal{B}(A, \mathbf{z}, d) \\
\text { for all } \ell \in \mathbb{Z}, i, j \in\{0, m\}
\end{array}\right\},
$$

and $\kappa(d)=\{(p, q):-d \leq p, q \leq d, p, q \in \mathbb{Z}\}$.

Proof. Without loss of generality, assume that $n$ is even and $s \leq n / 2$. Once $\mathcal{U}$ is given, construct $\mathcal{V}$ as above. Lemmas 3.7 and 3.9 indicate that $\mathbf{X}_{s}(\mathcal{V}) \cong$ $\mathbf{X}_{s}(\mathcal{U})$ and $\mathcal{V}$ is separable. Consider $d=n / 2$, Theorem 2.5 shows that there exists $(A, \mathbf{z}, d)$ so that $\mathcal{B}(A, \mathbf{z}, d)=\mathcal{V}$.

Let $m=2 d-s+1$. For each $x \in \mathbf{X}(\mathcal{B}(A, \mathbf{z}, d))$, (18) implies

$$
\begin{array}{r}
\pi_{\kappa(d)}\left(x_{\ell(i, j)}\right) \in \mathcal{B}(A, \mathbf{z}, d) \\
\text { for all } \ell \in \mathbb{Z}, \quad i, j \in\{0, n-s+1\} .
\end{array}
$$

It is easily seen that $\mathbf{X}(\mathcal{B}(A, \mathbf{z}, d))=\mathbf{X}_{s}(\mathcal{V})$. Since $\mathbf{X}_{s}(\mathcal{U}) \cong \mathbf{X}_{s}(\mathcal{V})$, the proof is completed.

When $(A, \mathbf{z}, d)$ is given, the basic set of admissible local patterns $\mathcal{B}=\mathcal{B}(A, \mathbf{z}, d)$ is immediately determined. Let $\Sigma_{p, q}(\mathbf{X}(\mathcal{B}))$ denote the set of global patterns in $\mathbf{X}(\mathcal{B})$ with size $p \times q$, and let $\Gamma_{p, q}(\mathbf{X}(\mathcal{B}))=\left|\Sigma_{p, q}(\mathbf{X}(\mathcal{B}))\right|$. The entropy of $\mathbf{X}(\mathcal{B})$ is defined by

$$
h(\mathbf{X}(\mathcal{B})) \equiv \lim _{p, q \rightarrow \infty} \frac{\log \Gamma_{p, q}(\mathbf{X}(\mathcal{B}))}{p q} .
$$

The existence of the limit can be found in [Chow et al., 1996b].

The first main theorem of this investigation, the dense entropy property of ICNN without input, is as follows.

Theorem A. For $t \in[0, \log 2], \varepsilon>0$, there exist $m \in \mathbb{N}$ and $(A, \mathbf{z}, d)$ such that $\mid h(\mathbf{X}(\mathcal{B}(A, \mathbf{z}, d)))$ $t \mid<\varepsilon$.

Before proving the theorem, the following lemmas should be stated first.

Lemma 3.11. Let $S_{n, l} \subset X^{n}$ be defined by

$$
\begin{aligned}
S_{n, l}= & \left\{x=\left(x_{1}, \ldots, x_{n}\right) \in X^{n}: x_{k}=-1\right. \\
& \text { for all } l+1 \leq k \leq n\},
\end{aligned}
$$

$1 \leq l \leq n-1$, and $S_{n, n}=X^{n}$. Then $S_{n, l}$ is separable.
Proof. Define a linear functional $g: \mathbb{R}^{n} \rightarrow \mathbb{R}$ by

$$
g(x)=\sum_{i=l+1}^{n} x_{i} \text { for all } x=\left(x_{i}\right)_{i=1}^{n} \in \mathbb{R}^{n} .
$$

Let $h(x)=g(x)+(n-l-1)$. It can be easily checked that $h(x)<0$ for all $x \in S_{n, l}$ and $h(x)>0$ for all $x \in S_{n, l}^{c}$. That is, $S_{n, l}$ and $S_{n, l}^{c}$ can be separated by the hyperplane

$$
H=\left\{x \in \mathbb{R}^{n}: g(x)=l-n+1\right\} .
$$

This completes the proof.

Theorem 3.12. Given $l, d \in \mathbb{N}$ and $n=4 d^{2}$. There exists $\mathcal{U}_{d, l} \subseteq\{1,-1\}^{\mathbb{Z}_{2 d \times 2 d}}$ such that $h\left(\mathbf{X}_{d}\left(\mathcal{U}_{d, l}\right)\right)=$ $(l / n) \log 2$ and $\mathcal{U}_{d, l}$ is separable, where $1 \leq l \leq n$.

Proof. If $d, l \in \mathbb{N}$ is given, $n=4 d^{2}$ and $1 \leq l \leq n$. Define

$$
T:\{1,-1\}^{\mathbb{Z}_{n \times 1}} \rightarrow\{1,-1\}^{\mathbb{Z}_{2 d \times 2 d}}
$$

by

$$
\begin{aligned}
(T \nu)_{i, j} & =\nu_{2 d(i-1)+j} \\
\text { for all } \nu & =\left(\nu_{k}\right) \in\{1,-1\}^{\mathbb{Z}_{n \times 1}} .
\end{aligned}
$$

Let $S_{n, l}$ be defined as in Lemma 3.11, and let $M_{n, l}$ be defined as follows.

$$
\begin{aligned}
& M_{n, l} \equiv\left\{K \in\{1,-1\}^{\mathbb{Z}_{2 d \times 2 d}}: \exists \nu \in S_{n, l}\right. \\
&\text { such that } K=T \nu\} .
\end{aligned}
$$

Furthermore, construct $\mathcal{U}_{d, l} \subseteq\{1,-1\}^{\mathbb{Z}_{4 d \times 4 d}}$ as follows. $J \in \mathcal{U}_{d, l}$ if $\pi_{(i, j)+\Lambda(2 d)}(J) \in M_{n, l}$ for $i, j \in$ $\{0,2 d\}$, where $\Lambda(n)$ is defined as in Definition 3.1.

Claim. $\mathcal{U}_{d, l}$ is separable.

Let $g: \mathbb{R}^{n} \rightarrow \mathbb{R}$ be defined as in (20) and $\tilde{g}=g \circ T^{-1}$. For $w \in\{1,-1\}^{\mathbb{Z}_{4 d \times 4 d}}$, rewriting $w$ as $w=\begin{array}{ll}w_{1} & w_{2} \\ w_{3} & w_{4}\end{array}$, where $w_{i} \in\{1,-1\}^{\mathbb{Z}_{2 d \times 2 d}}$ for all $i$. Define a linear functional $\tau:\{1,-1\}^{\mathbb{Z}_{4 d \times 4 d}} \rightarrow \mathbb{R}$ by $\tau(w)=\tilde{g}\left(w_{1}\right)+\tilde{g}\left(w_{2}\right)+\tilde{g}\left(w_{3}\right)+\tilde{g}\left(w_{4}\right)$ and $\tilde{\tau}(w)=\tau(w)+4 n-4 l-1$. The above constitution confirms that $\tilde{\tau}(w)<0$ for all $w \in \mathcal{U}_{d, l}$ and $\tilde{\tau}(w)>0$ otherwise. That means $\mathcal{U}_{d, l}$ is separable. 
Moreover, there are $2^{l}$-many patterns for each block of $x \in \mathbf{X}_{d}\left(\mathcal{U}_{d, l}\right)$ with size $2 d \times 2 d$. Therefore,

$$
\begin{aligned}
h\left(\mathbf{X}_{d}\left(\mathcal{U}_{d, l}\right)\right) & =\lim _{p, q \rightarrow \infty} \frac{\log \Gamma_{2 d p \cdot 2 d q}\left(\mathbf{X}_{d}\left(\mathcal{U}_{d, l}\right)\right)}{2 d p \cdot 2 d q} \\
& =\lim _{p, q \rightarrow \infty} \frac{\log \left(2^{l}\right)^{p q}}{4 d^{2} p q} \\
& =\frac{l}{n} \log 2 .
\end{aligned}
$$

This completes the proof.

Proof of Theorem A. For $t \in[0, \log 2]$ and $\varepsilon>0$, there exist $d, l \in \mathbb{N}$ such that $|(l / n) \log 2-t|<$ $\varepsilon$, where $n=4 d^{2}$. Theorem 3.12 indicates there is a separable set $\mathcal{U}_{d, l}$ such that $h\left(\mathbf{X}_{d}\left(\mathcal{U}_{d, l}\right)\right)=$ $(l / n) \log 2$. Lemma 3.7, Lemma 3.9 and Theorem 3.10 show $h\left(\mathbf{X}_{d}\left(\mathcal{U}_{d, l}\right)\right)=h\left(\mathbf{X}_{d}\left(\mathcal{V}_{d, l}\right)\right)$ and there exist $m \in \mathbb{N}$ and $(A, \mathbf{z}, d)$ such that $\mathcal{B}(A, \mathbf{z}, d)=\mathcal{V}_{d, l}$. The proof is then completed.

\section{Inhomogeneous Cellular Neural Networks with Input}

In this section, Theorem A is extended to the case where $B$ is not identical to zero.

Once the parameters $(A, B, \mathbf{z}, d)$ are given, the basic set of admissible local patterns is determined and denoted by

$$
\begin{aligned}
\mathcal{B} & \equiv \mathcal{B}(A, B, \mathbf{z}, d) \\
& =\{Y \circ U\} \subseteq\{1,-1\}^{\mathbb{Z}_{(2 d+1) \times(2 d+1) \times 2},}
\end{aligned}
$$

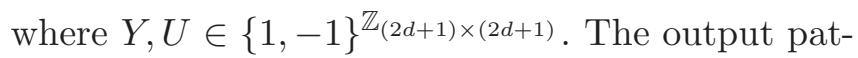
tern $Y$ coupled with input pattern $U$, denoted by $Y \circ U$, is a two-layer array. Defining the output space generated by $\mathcal{B}(A, B, \mathbf{z}, d)$ as follows.

$$
\mathbf{X}(\mathcal{B})=\left\{\begin{array}{c}
y \in\{1,-1\}^{\mathbb{Z}^{2}}: \text { there exists } u \in\{1,-1\}^{\mathbb{Z}^{2}} \text { such that } \\
\pi_{\kappa(d)}\left(\sigma_{(i, j)}^{\ell}(y \circ u)\right) \in \mathcal{B} \text { for all } \ell \in \mathbb{Z}, i, j \in\{0, m\}
\end{array}\right\},
$$

where $\kappa(d)$ is defined in Theorem 3.10 and

$\pi_{\kappa(d)}\left(\sigma_{(i, j)}(y \circ u)\right) \equiv \pi_{\kappa(d)}\left(\sigma_{(i, j)}(y)\right) \circ \pi_{\kappa(d)}\left(\sigma_{(i, j)}(u)\right)$.

For $d, l \in \mathbb{N}$, let $\mathcal{U}_{d, l}$ be the same as defined in the proof of Theorem 3.12. Denote by

$$
\mathcal{V}_{d, l}=\left\{Y \circ U: Y, U \in \mathcal{U}_{d, l}\right\} \subseteq\{1,-1\}^{\mathbb{Z}_{2 d \times 2 d \times 2}} .
$$

Then the lemma follows.

Lemma 4.1. $\mathcal{V}_{d, l}$ is separable.

Proof. Let $\tau$ be the same as in the proof of Theorem 3.12. Define a linear functional $\theta:\{1,-1\}^{\mathbb{Z}_{4 d \times 4 d}} \times$

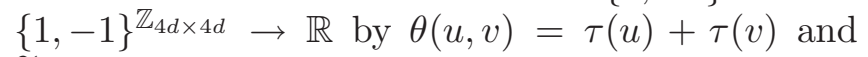
$\widetilde{\theta}(u, v)=\theta(u, v)+8 n+8 l-1$. It is easily checked that $\widetilde{\theta}(u \circ v)<0$ for all $u \circ v \in \mathcal{V}_{d, l}$ and $\widetilde{\theta}(u \circ v)>0$ otherwise. This completes the proof.

Furthermore, the entropy of the subshift space induced by $\mathcal{V}_{d, l}$ can be computed via the same method as in the proof of Theorem 3.12, thus the proof is omitted.

Theorem 4.2. $h\left(\mathbf{X}_{d}\left(\mathcal{V}_{d, l}\right)\right)=(l / n) \log 2$.

The dense entropy property of ICNN with input then follows.
Theorem B. For $t \in[0, \log 2], \varepsilon>0$, there exist $m \in \mathbb{N}$ and $(A, B, \mathbf{z}, d)$ such that $\mid h(\mathbf{X}(\mathcal{B}(A, B$, $\mathbf{z}, d)))-t \mid<\varepsilon$.

The proof of Theorem B can be accomplished via the same discussion in the proof of Theorem A, hence is skipped.

\section{Acknowledgment}

The authors thank Prof. Song-Sun Lin for some helpful discussions and suggestions.

\section{References}

-Ban, J.-C., Chien, K.-P. \& Lin, S.-S. [2001a] "Spatial disorder of CNN-with asymmetric output function," Int. J. Bifurcation and Chaos 11, 2085-2095.

-Ban, J.-C., Lin, S.-S. \& Shih, C.-W. [2001b] "Exact number of mosaic patterns in one-dimensional cellular neural networks," Int. J. Bifurcation and Chaos 11, $1645-1653$.

-Ban, J.-C., Hsu, C.-H. \& Lin, S.-S. [2002] "Spatial disorder of cellular neural network-with biased term," Int. J. Bifurcation and Chaos 12, 525-534.

-Ban, J.-C. \& Lin, S.-S. [2005] "Patterns generation and transition matrices in multi-dimensional lattice models," Discr. Contin. Dyn. Syst. 13, 637-658. 
- Ban, J.-C., Lin, S.-S. \& Lin, Y.-H. [2007a] "Patterns generation and spatial entropy in two-dimensional lattice models," Asian J. Math. 11, 497-534.

Ban, J.-C., Chang, C.-H., Lin, S.-S. \& Lin, Y.-H. [2007b] "Entropy and zeta function for one-dimensional multi-layer cellular neural networks," preprint.

-Chow, S.-N., Mallet-Paret, J. \& Van Vleck, E. S. [1996a] "Dynamics of lattice differential equations," Int. J. Bifurcation and Chaos 6, 1605-1621.

Chow, S.-N., Mallet-Paret, J. \& Van Vleck, E. S. [1996b] "Pattern formation and spatial chaos in spatially discrete evolution equations," Rand. Comput. Dynam. 4, 109-178.

-Chua, L.-O. \& Yang, L. [1988a] "Cellular neural networks: Theory," IEEE Trans. Circuits Syst. 35, $1257-1272$.

-Chua, L.-O. \& Yang, L. [1988b] "Cellular neural networks: Applications," IEEE Trans. Circuits Syst. 35, 1273-1290.

Chua, L.-O. [1998] CNN: A Paradigm for Complexity, World Scientific Series on Nonlinear Science, Series A, Vol. 31 (World Scientific, Singapore).

-Debye, P. \& Bueche, A. M. [2004] "Scattering by an inhomogeneous solid," J. Appl. Phys. 20, 518-525.

-Desai, A. [2006] "Subsystem entropies for $\mathbb{Z}^{d}$ sofic systems," Indagationes Mathematicae 17, 353-359.

-Fäth, M., Freisem, S., Menovsky, A. A., Tomioka, Y., Aarts, J. \& Mydosh, J. A. [1999] "Spatially inhomogeneous metal-insulator transition in doped manganites," Science 285, 1540-1542.

-Ferdinand, A. E. \& Fisher, M. E. [1969] "Bounded and inhomogeneous ising models. I. Specific-heat anomaly of a finite lattice," Phys. Rev. 185, 832-846.

-Hsu, C.-H., Juang, J., Lin, S.-S. \& Lin, W.-W. [2000] "Cellular neural networks: Local patterns for general template," Int. J. Bifurcation and Chaos 10, 16451659 .
-Hsu, C.-H. \& Yang, T.-H. [2002] "Abundance of mosaic patterns for CNN with spatially variant templates," Int. J. Bifurcation and Chaos 12, 1321-1332.

- Juang, J. \& Lin, S.-S. [2000] "Cellular neural networks: Mosaic pattern and spatial chaos," SIAM J. Appl. Math. 60, 891-915.

Kravtsov, Y. A. \& Orlov, Y. I. [1990] Geometrical Optics of Inhomogeneous Media (Springer-Verlag, Berlin Heidelberg, NY).

Lay, R. [1992] Convex Sets and Their Applications (Wiley, NY).

-Lin, S.-S. \& Shih, C.-W. [1999] "Complete stability for standard cellular neural networks," Int. J. Bifurcation and Chaos 9, 909-918.

-Lin, S.-S. \& Yang, T.-S. [2000] "Spatial entropy of onedimensional celluar neural network," Int. J. Bifurcation and Chaos 10, 2129-2140.

-Lin, S.-S. \& Yang, T.-S. [2002] "On the spatial entropy and patterns of two-dimensional cellular neural networks," Int. J. Bifurcation and Chaos 12, 115-128.

Lind, D. \& Marcus, B. [1995] An Introduction to Symbolic Dynamics and Coding (Cambridge University Press, Cambridge).

-Perdew, J. P. [1986] "Density-functional approximation for the correlation energy of the inhomogeneous electron gas," Phys. Rev. B 33, 8822-8824.

Pesin, Y. [1997] Dimension Theory in Dynamical Systems (University of Chicago Press).

-Quas, A. \& Trow, P. [2000] "Subshifts of multidimensional shifts of finite type," Erg. Th. Dyn. Syst. 20, 859-874.

-Quas, A. \& Sahin, A. [2003] "Entropy gaps and locally maximal entropy in $\mathbb{Z}_{d}$ subshifts," Erg. Th. Dyn. Syst. 23, $1227-1245$.

- Rosenfeld, Y. [1989] "Free-energy model for the inhomogeneous hard-sphere fluid mixture and densityfunctional theory of freezing," Phys. Rev. Lett. 63, 980-983. 